\title{
Development of a canine model of pulmonary emphysema and imaging of the emphysematous lung with infrared thoracoscopy
}

\author{
Masashi Gotoh, MD \\ Taku Okamoto, MD \\ Yasumichi Yamamoto, MD \\ Dage Liu, MD \\ Kotaro Kameyama, MD \\ Eiichi Hayashi, MD \\ Cheng-Long Huang, MD \\ Hiroyasu Yokomise, MD
}

Background: The surgeon must use the results of preoperative computed tomography findings and scintigraphic studies to make a subjective decision during lung volume reduction surgery with regard to the best incision line.

Objectives: Our purpose was to develop an objective and real-time method of detecting areas of pulmonary emphysema by using infrared thoracoscopy.

Methods: Pulmonary emphysema was developed in various lobes of the lung in the dog by injecting porcine pancreatic elastase through the bronchi. One month after the injection, chest computed tomography and lung biopsy were performed. Infrared thoracoscopic study was then performed to reveal the presence of the emphysematous lung tissue. Simultaneously, indocyanine green was injected intravenously, and the time taken for each type of lung tissue to stain blue was measured.

Results: Chest computed tomography and histologic examinations revealed pulmonary emphysema-like areas in the porcine pancreatic elastase-injected lung. The computed tomography numbers of the porcine pancreatic elastase-injected lung tissue, normal lung tissue, and the tracheal lumen were $-868.8 \pm 18.6,-752.2 \pm$ 32.5 , and $-1013 \pm 27.1$, respectively. There were significant differences between the porcine pancreatic elastase-injected lung and the normal lung $(P<.0001)$. The time for staining to begin was $10.7 \pm 4.8$ seconds for normal lung tissue and 25.8 \pm 9.4 seconds for the emphysematous tissue; the onset of staining emphysematous lung tissue was significantly delayed $(P=.003)$.

Conclusions: We developed a successful canine model of pulmonary emphysema by injecting porcine pancreatic elastase. Infrared thoracoscopic examination revealed that the staining of emphysematous lung using indocyanine green injection was significantly greater than of normal lung.

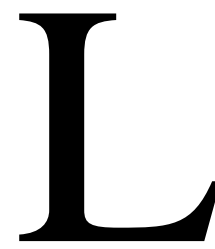

ung volume reduction surgery (LVRS) has recently become a common procedure for the surgical treatment of pulmonary emphysema. ${ }^{1}$ This procedure reduces the lung volume by excising over-expanded emphysematous tissue to increase lung elasticity and contraction, improve thoracic compliance, and improve physical ability and symptoms of dyspnea. ${ }^{1}$ The surgeon uses his or her experience to determine the best line of excision from examination of preoperative chest computed tomography (CT) and scintigraphic studies, together with the intraoperative 


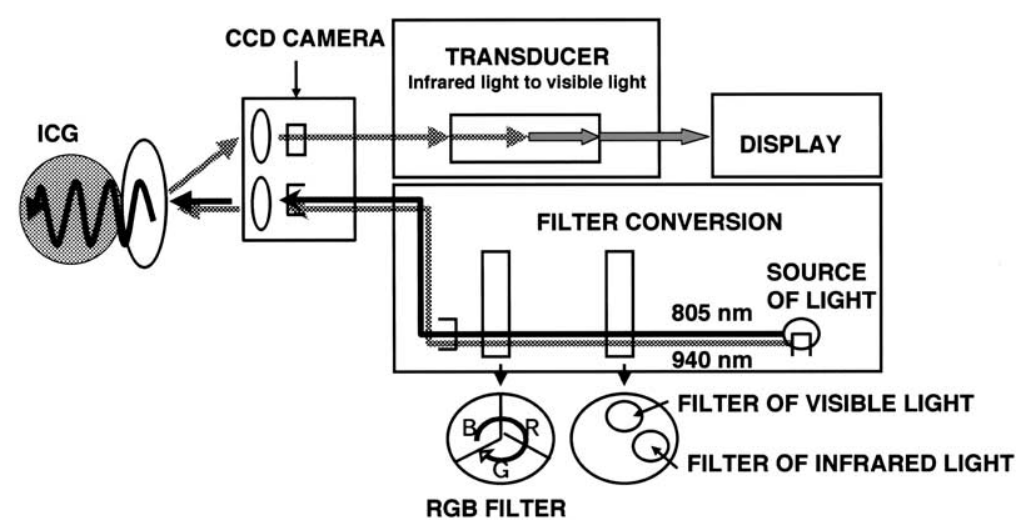

Figure 1. Infrared thoracoscopy display system.

macroscopic appearance of the affected region. The development of a method for differentiating emphysematous and normal lung tissue in a real-time and quantitative manner would increase the accuracy of selection of the incision line and lead to optimum reduction of lung volume.

In addition, it has been reported that the recurrence rate of pneumothorax can be as high as $10 \%$ when thoracoscopic bullectomy is selected as a treatment method. ${ }^{2-4}$ In contrast, the recurrence rate is approximately $1 \%$ to $2 \%$ when thoracotomy is performed for bullectomy in a similar situation. ${ }^{2-4}$ This difference in pneumothorax recurrence rates is believed to be partly caused by the fact that bullae tend to be easily overlooked during thoracoscopic surgery because of the presence of a flaccid lung during single lung ventilation. ${ }^{2-4}$ Detection of small or flaccid bullae that cannot be recognized macroscopically greatly decreases the postoperative occurrence of pneumothorax.

Infrared thoracoscopy that can detect 2 different wavelengths of infrared light can display indocyanine green (ICG) with an absorption peak in the infrared light region $(805 \mathrm{~nm})$ as blue. We induced emphysema in the lung of a dog and observed and imaged the emphysematous lung with infrared thoracoscopy in accordance with the principle that the emphysematous lung has a reduced blood flow.

Our objectives were to develop a canine model of pulmonary emphysema and to identify emphysematous areas of the lung by using infrared thoracoscopy.

\section{Methods and Materials}

\section{Experiment 1: Development of the Canine Model of Pulmonary Emphysema}

Porcine pancreatic elastase (PPE, $350 \mathrm{U} / \mathrm{kg}$ body weight; Elastin Products Co, Owensville, Mo) was dissolved in distilled water (1 $\mathrm{mL} / \mathrm{kg}$ body weight). Seven beagle dogs (body weight $10-12 \mathrm{~kg}$, mean body weight $10.7 \mathrm{~kg}$ ) were anesthetized by intramuscular injection of xylazine (Selactal, $5.0 \mathrm{mg} / \mathrm{kg}$; Bayer Ltd, Tokyo, Japan) and ketamine (Ketalar, $125 \mathrm{mg} / \mathrm{kg}$; Sankyo Co, Ltd, Tokyo, Japan). After introducing a bronchoscope (Olympus: 1T40, Tokyo,
Japan) into the bronchi, we inserted a Swan-Ganz catheter (7F; Edwards Lifesciences, Irvine, Calif) into the segmental bronchus through the channel of the bronchoscope under mechanical ventilation. The injected lung lobes were selected randomly as follows: right upper lobe in $2 \mathrm{dogs}$, right lower lobe in $1 \mathrm{dog}$, left upper lobe in 2 dogs, and left lower lobe in 2 dogs. PPE solution was injected selectively into each lobe through the catheter. After the PPE solution had been injected and the balloon of the Swan-Ganz catheter had been blown up, the opening of the bronchus through which the PPE had been injected was blocked for 15 minutes to prevent backflow of PPE. Four weeks after injection, the PPEinjected lungs and normal lungs were observed histologically. At this time, chest CT was performed to obtain radiologic images in all animals. The CT numbers of the PPE-injected lobes, normal lobes, and the tracheal lumen were determined at 6 points in each area in a random manner.

\section{Experiment 2: Observation of the Normal Lung and Emphysematous Lung with Infrared Thoracoscopy}

Infrared thoracoscopy. Infrared thoracoscopy uses an instrument that irradiates an infrared light (805 $\mathrm{nm}$ and $940 \mathrm{~nm}$ ) from a light source, receives reflected light with a charge coupled device camera, and displays the data on the monitor after signal conversion. ${ }^{5}$ Infrared light at $805 \mathrm{~nm}$ is displayed as red and green components on the monitor, and infrared light at $940 \mathrm{~nm}$ is displayed as a blue component. Any object that reflects all colors is displayed as white because all 3 colors are mixed, and objects that reflect little light at $805 \mathrm{~nm}$ are displayed in blue because only light reflected light at $940 \mathrm{~nm}$ is detected. ICG is displayed in blue (Figures 1 and 5).

A 22 GA plastic catheter (Angiocath; Becton Dickinson Infusion Therapy Systems Inc, Franklin Lakes, NJ) containing ICG was inserted into the removed lung. The collapsed lung and catheter with ICG were observed with infrared thoracoscopy. By the insertion angle of the catheter, it was possible to know how deeply infrared thoracoscopy can observe lung parenchyma.

Observation of the normal lung. The lung of a control dog (12 $\mathrm{kg}, \mathrm{n}=1$ ) was observed under normal light and infrared light by using thoracoscopy. We injected $25 \mathrm{mg}$ of ICG rapidly into the forearm vein for observation of the lung under infrared light. The 


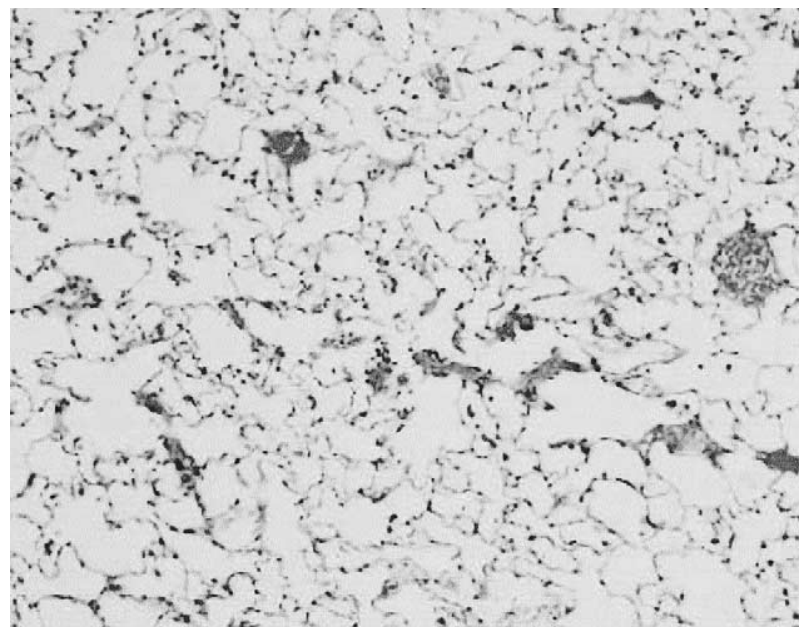

Figure 2. Histologic appearance of the normal dog lung is similar to that of the normal human lung (hematoxylin-eosin stain, $\times 40$ ).

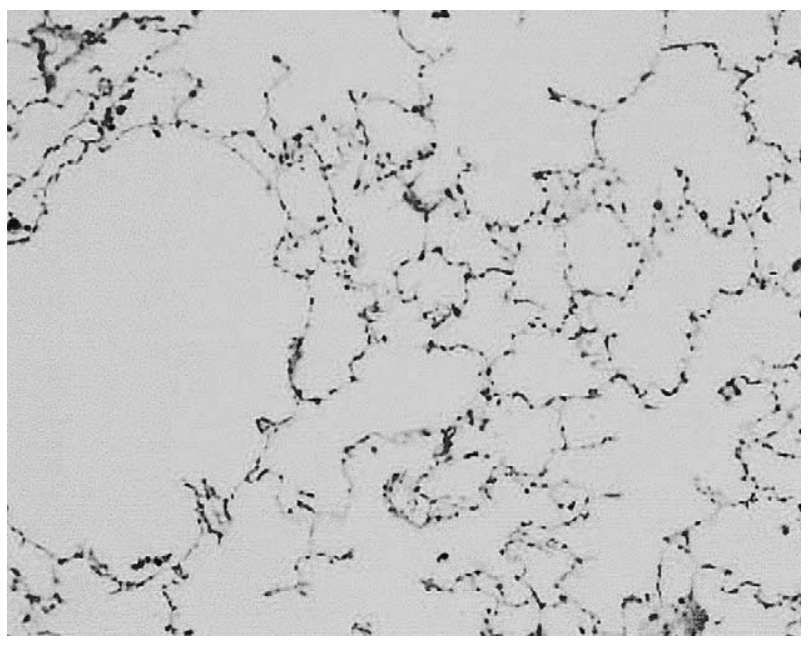

Figure 3. Histologic examination of porcine pancreatic elastaseinjected lung tissue showed that the alveolar structure was destroyed and pulmonary emphysema was present (hematoxylin and eosin stain, $\times 40$ ).

infrared thoracoscope used was an Olympus infrared endoscope (XBF-200IR). After induction of anesthesia with an intramuscular injection of xylazine $(5.0 \mathrm{mg} / \mathrm{kg})$ and ketamine $(125 \mathrm{mg} / \mathrm{kg})$, a Univent endobronchial tube (Fuji Systems Co, Tokyo, Japan) was introduced for bronchoscopy under anesthesia with inhaled sevoflurane. A trocar was inserted under single-lung ventilation, and the lung was observed under normal light and infrared light by thoracoscopy.

Observation of emphysematous lung with infrared thoracoscopy. Four weeks after injection of PPE into the adult beagle dogs $(10-12 \mathrm{~kg} ; \mathrm{n}=7)$, their lungs were observed with the normal light and the infrared thoracoscope. We then rapidly injected $25 \mathrm{mg}$ of ICG into the forearm vein to observe the lungs under infrared light. After observation, chest CT was performed, and then all dogs were killed for histologic examination.

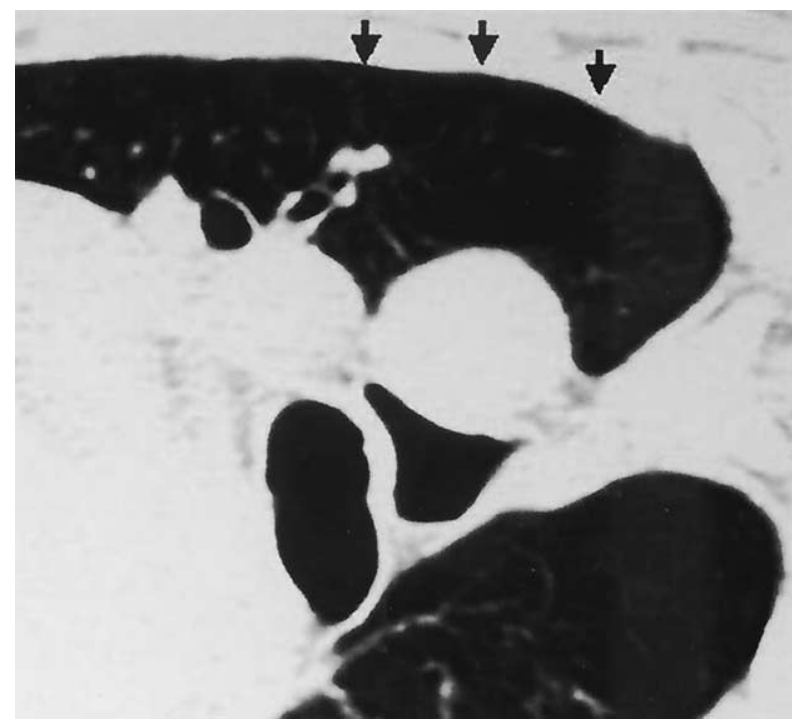

Figure 4. Chest computed tomography showing pulmonary emphysema (arrows) induced by PPE injection.

All the animal experiments were performed in accordance with the "Guide for the Care and Use of Laboratory Animals" prepared by the Institute of Laboratory Animal Resources and published by the National Institutes of Health (publication No. 86-23, revised 1985)

\section{Results}

Development of the Pulmonary Emphysema Model We injected PPE into all 7 dogs. The histologic appearance of the normal lung is shown (Figure 2). The normal lung tissue had an alveolar structure similar to that of normal human lung. All 7 experimental dogs had macroscopically hyperexpanded and less flaccid emphysematous changes in the PPE-injected lobes. The histologic appearance of the PPE-injected lobes is shown (Figure 3). The alveolar structure had been destroyed, and histologic evidence of pulmonary emphysema was clearly apparent. Chest CT revealed findings consistent with those of pulmonary emphysema in the area of the injection (Figure 4). The CT numbers of PPE-injected lung tissue, normal lung, and the tracheal lumen were $-868.8 \pm 18.6,-752.2 \pm 32.5$, and $-1013 \pm$ 27.1 , respectively. There were significant differences between the CT numbers of the PPE-injected lung tissue and the normal lung tissue $(P<.0001)$ (Table 1$)$. The CT numbers of the PPE-injected lung tissue indicated a density more similar to that of air than that of normal lung tissue.

\section{Observation of the Normal Lung with Infrared Thoracoscopy}

The observation of the catheter containing ICG revealed that infrared thoracoscopy could observe normal lung parenchyma $0.2 \mathrm{~cm}$ in depth. This depth seems to change 
TABLE 1. CT numbers of PPE-injected lung, normal lung, and tracheal lumen

$\left.\begin{array}{lc}\hline & \multicolumn{1}{c}{\text { CT number }} \\ \hline \text { PPE-injected lung } & -868 \pm 18.6 \\ \text { Normal lung } & -752 \pm 32.5 \\ \text { Tracheal lumen } & -1013 \pm 27.1\end{array}\right]+$

$C T$, Computed tomography; $P P E$, porcine pancreatic elastase.

${ }^{*}, \dagger P<.0001$, mean \pm SD.

according to the state of the lung. We rapidly injected ICG into the forearm vein of the normal control dog to observe the thoracic cavity under infrared light. Blue staining began after approximately 6 seconds and reached a peak 8 seconds after the intravenous injection (Figure 5). Infrared thoracoscopy after ICG injection gave images that were strongly blue in areas of atelectasis. During the 10-minute observation period, the intensity of the blueness diminished slightly but remained easily distinguishable.

\section{Observation of the Pulmonary Emphysema Model Lung with Infrared Thoracoscopy}

The conventional thoracoscopic (white light thoracoscopy) examination failed to detect the emphysematous area. With infrared thoracoscopy, there were differences between the PPE-injected lobes and normal lobes of the 7 experimental dogs in the time of onset of blue staining after intravenous ICG injection. Pulmonary lobes not injected with PPE began to stain blue in $10.7 \pm 4.8$ seconds. In contrast, lobes injected with PPE began to stain blue in $25.8 \pm 9.4$ seconds; in the injected lobes there were significant delays in the onset of blue staining compared with the uninjected lobes $(P$ $=.003)$. In addition, when we compared both areas histologically, damage to the alveolar structure and emphysematous changes were clearly apparent in the areas of delayed blue staining (Figure 6).

\section{Discussion}

We developed a successful canine model of pulmonary emphysema by injecting PPE inside the bronchi. Various methods of development of the pulmonary emphysema model have been reported. ${ }^{6-10}$ However, we chose the method of bronchial injection of $\mathrm{PPE}^{11}$ because it was simple and reliable. Evaluation of pulmonary emphysema was performed histopathologically, and damage to the alveolar structure was observed. In addition, chest CT revealed features of pulmonary emphysema in the injected lungs. There were significant differences between the CT numbers of PPE-injected lung tissue and those of normal lung tissue. The CT numbers of the PPE-injected lungs indicated a density more similar to that of air than did those of the uninjected lungs. From these results, we consistently confirmed the successful induction of pulmonary emphysema-like lesions in dogs through PPE injection.
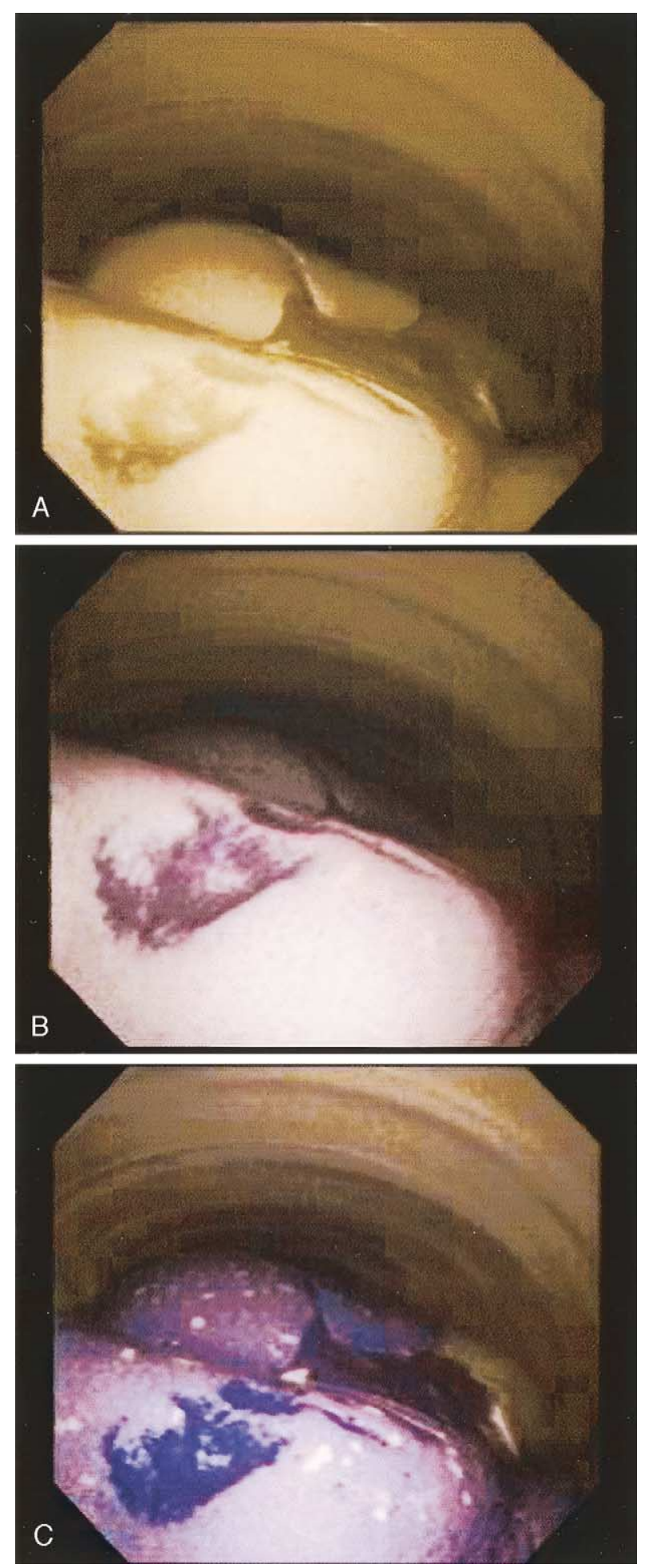

Figure 5. Infrared thoracoscopic findings in the normal lung tissue of the control dog before indocyanine green (ICG) injection (A), 6 seconds after ICG injection (B), and 8 seconds after ICG injection (C). Blue staining of the lung began approximately 6 seconds after intravenous injection, and the staining peaked at 8 seconds. After ICG intravenous injection, areas of atelectasis appeared as a rich, strong blue. 


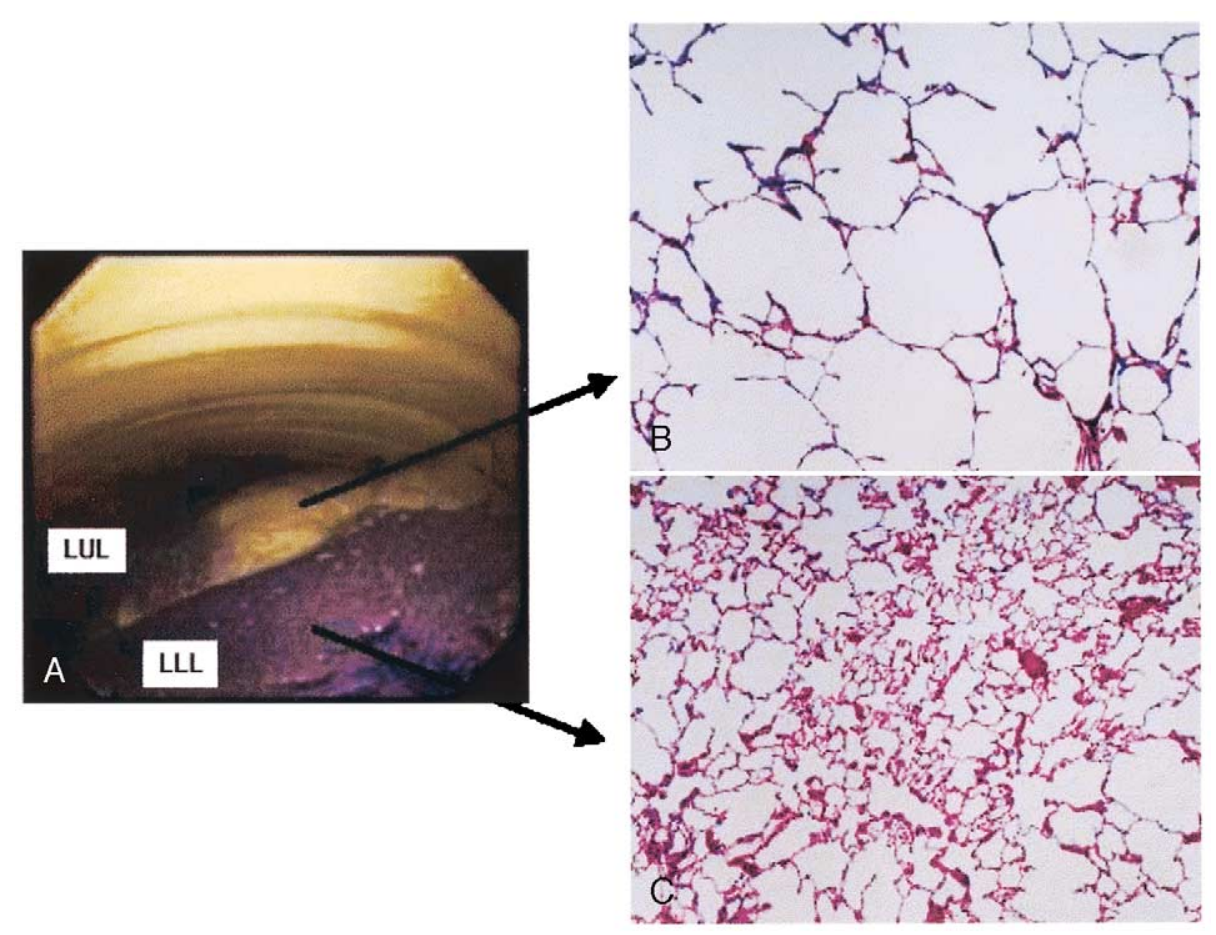

Figure 6. Thoracoscopic and histologic findings in the emphysematous lung. A, Thoracoscopy revealed areas of faster and slower onset of blue staining. $B$, Histologic examination revealed a damaged alveolar structure in the area of slower onset of staining (hematoxylin and eosin stain, $\times 40$ ). C, Histologic examination revealed an alveolar structure similar to that of the normal human lung (hematoxylin and eosin stain, $\times 40$ ).

In our observation of the normal lung by infrared thoracoscopy, all lobes began to stain blue simultaneously approximately 6 seconds after the intravenous injection of ICG. Areas of atelectasis were stained dark blue. ICG has an absorption peak of approximately $805 \mathrm{~nm},{ }^{12}$ and it appears blue when there is weak reflection of light with a wavelength of approximately $805 \mathrm{~nm}$. This weak reflection occurs because intravenously injected ICG spreads in the bloodstream and is distributed over the lung. ${ }^{13}$ Regions of atelectasis appear as an intense blue because they are less well pneumatized than inflated lung tissue and are thus highly dense, with a higher blood flow. The intensity of the blue staining is thus determined by the amount of blood flow in the area. Lobes in which emphysema was induced by PPE injection had a significantly delayed onset of blue staining compared with uninjected lobes. In addition, areas that showed delayed onset of blue staining were histologically determined to be highly emphysematous. Therefore, we believe that the tissue density of the emphysematous lung was lower than that of normal lung because damage to the alveolar structure led to expansion of the alveolar cavity; consequently, a difference in the intensity of the blue staining was created as the blood flow decreased. Our observation that areas displaying a delayed onset of blue staining were histologically areas of pulmonary emphysema indi- cates that the administration of ICG under infrared thoracoscopy allowed us to distinguish emphysematous lung tissue from normal lung tissue. In a clinical situation, the target area in LVRS is not surrounded by normal lung tissue but by less emphysematous tissue. We must try the clinical examination in a trial and error manner.

By using this method, it will be possible to identify areas with highly emphysematous changes and to select an appropriate incision line for LVRS. Furthermore, this method will be useful for finding small bullae and blebs that might otherwise have been overlooked during video-assisted thoracoscopic surgery for spontaneous pneumothorax.

\section{References}

1. Cooper JD, Trulock EP, Triantafillou AN, Patterson GA, Pohl MS, Deloney PA, et al. Bilateral pneumectomy (volume reduction) for chronic obstructive pulmonary disease. J Thorac Cardiovasc Surg. 1995;109:106-16.

2. Horio H, Nomori H, Fuyuno G, Kobayashi R, Suemasu K. Limited axillary thoracotomy vs video-assisted thoracoscopic surgery for spontaneous pneumothorax. Surg Endosc. 1998;12:1155-8.

3. Kim KH, Kim HK, Han JY, Kim JT, Won YS, Choi SS. Transaxillary minithoracotomy versus video-assisted thoracic surgery for spontaneous pneumothorax. Ann Thorac Surg. 1996;61:1510-2.

4. Cole FH Jr, Cole FH, Khandekar A, Maxwell JM, Pate JW, Walker WA. Video-assisted thoracic surgery: primary therapy for spontaneous pneumothorax? Ann Thorac Surg. 1995;60:931-5. 
5. Tanimizu M, Itoshima T, Ukida M, Ito T, Yamamoto K, Tanaka R, et al. Infrared peritoneoscopy of the river using Visicon camera and indocyanine green. Yakuri to Chiryo. 1988;16:131-41.

6. Chen JC, Brenner M, Kafie FE, Yoong B, Budd M, Gassel A, et al. An animal model for lung volume reduction therapy of pulmonary emphysema. Invest Surg. 1998;11:129-37.

7. Akahane T, Kurokawa Y, Chiba R, Yaegashi H, Takahashi T, Satomi S. Effects of Nd:YAG laser irradiation on morphometry and lung function in elastase-induced emphysema in rats. Lasers Surg Med. 1998;23:204-12.

8. Suga T, Kurabayashi M, Sando Y, Ohyama Y, Maeno T, Aizawa H, et al. Disruption of the klotho gene causes pulmonary emphysema in mice. Defect in maintenance of pulmonary integrity during postnatal life. Am J Respir Cell Mol Biol. 2000;22:26-33.
9. Snider GL, Lucey EC, Stone PJ. Animal models of emphysema. Am Rev Respir Dis. 1986;133:149-69.

10. Hautamaki RD, Kobayashi DK, Senior RM, Shapiro SD. Requirement for macrophage elastase for cigarette smoke-induced emphysema in mice. Science. 1997;277:2002-4.

11. Noma S, Herman PG, Khan A, Rojas KA, Pipman Y. Sequential morphologic changes of elastase-induced pulmonary emphysema in pig lungs. Evaluation by high-resolution computed tomography. Invest Radiol. 1991;26:446-53.

12. Greenwell TJ, Wyman A, Rogers K. Chromophore-enhanced $805 \mathrm{~nm}$ laser therapy for gastrointestinal neoplasia. Eur J Surg Oncol. 2001; 27:368-72.

13. Kohso H. Fundamental and clinical studies on the infrared ray electronic endoscopy. Kyoto Fritsu Idai Shi. 1993;102:343-65. 